Advances in Dynamical Systems and Applications

ISSN 0973-5321, Volume 14, Number 2, pp. 201-211 (2019)

https://dx.doi.org/10.37622/ADSA/14.2.2019.201-211

\title{
Positive Solutions to Singular Second-Order Boundary Value Problems on Time Scales
}

\author{
Curtis Kunkel \\ University of Tennessee Martin \\ Department of Mathematics \& Statistics \\ Martin, Tennessee, 38238, USA
}

\begin{abstract}
We study singular second order boundary value problems with mixed boundary conditions on general time scales. We prove the existence of a positive solution by means of a lower and upper solutions method and the Brouwer fixed point theorem, in conjunction with perturbation methods used to approximate regular problems.
\end{abstract}

AMS Subject Classifications: 34B16, 34B18, 34B40, 39A10.

Keywords: Singular boundary value problems, time scales, mixed conditions, lower and upper solutions, Brouwer fixed point theorem, approximate regular problems.

\section{Introduction}

This paper concludes the work done previously on second-order boundary value problems by Kunkel $[8,9]$, where he studied a second-order singular boundary value problem for differential equations and a second-order singular boundary value problem on purely discrete time scales of nonuniform step size, respectively. Although similar throughout most of the time scale, this result is different in the fact that the time scale itself is entirely general, meaning that it could be entirely continuous (as in [8]), could be entirely discrete (as in [9]), or could be any combination along this continuum so long as the underlying interval remains a closed subset of $\mathbb{R}$.

More specifically, [8] dealt with the singular boundary value problem along a continuous interval (i.e. a standard differential equation),

$$
\begin{gathered}
u^{\prime \prime}(t)+f(t, u(t))=0, \quad t \in(0,1), \\
u^{\prime}(0)=u(1)=0,
\end{gathered}
$$

Received March 26, 2019; Accepted May 3, 2019

Communicated by John Graef 
where $f(t, x)$ is singular in $x$. Similar methods to what are introduced in this paper are used to prove the existence of a positive solution.

Alternatively, [9] dealt with the singular boundary value problem along a discrete interval with nonuniform step size (i.e. a difference equation with graininess not necessarily equal to 1$)$,

$$
\begin{gathered}
u^{\Delta \Delta}\left(t_{i-1}\right)+f\left(t_{i}, u\left(t_{i}\right), u^{\Delta}\left(t_{i-1}\right)\right)=0, \quad t \in \mathbb{T}^{\circ}, \\
u^{\Delta}\left(t_{0}\right)=u\left(t_{n+1}\right)=0,
\end{gathered}
$$

where $\mathbb{T}^{\circ}$ is the discrete interval of nonuniform step size $\left[t_{1}, t_{n}\right]:=\left\{t_{1}, t_{2}, \ldots, t_{n}\right\}$ and $f(t, x, y)$ is singular in $x$. This work was an extension of a previous result by Rachůnková and Rachůnek [12], where they studied a second-order singular boundary value problem for the discrete $p$-Laplacian, $\phi_{p}(x)=|x|^{p-2} x, \quad p>1$. In particular, Rachůnková and Rachůnek dealt with the discrete boundary value problem,

$$
\begin{gathered}
\Delta\left(\phi_{p}(\Delta u(t-1))\right)+f(t, u(t), \Delta u(t-1))=0, \quad t \in[1, T+1], \\
\Delta u(0)=u(T+2)=0,
\end{gathered}
$$

in which $f(t, x, y)$ was singular in $x$.

This result melds these two ends of the time scale continuum creating a unifying theorem for this type of problem across all types of time scales. The methods in this paper rely heavily on lower and upper solution methods in conjunction with an application of the Brouwer fixed point theorem [14]. We consider only the singular second-order boundary value problem, while letting our function range over a time scale with specified beginning and ending values, using these in defining our boundary conditions. We will provide some definitions from the Bohner and Peterson text on time scales, [4], along with specific definitions of appropriate lower and upper solutions. The lower and upper solutions will be applied to nonsingular perturbations of our nonlinear problem, ultimately giving rise to our boundary value problem by passing to the limit.

Lower and upper solutions have been used extensively in establishing solutions of boundary value problems for finite difference equations. Representative works include $[3,7,11]$. This method is also well used in establishing solutions of boundary value problems for ordinary differential equations. Representative works include $[2,5,6,10]$.

Singular boundary value problems have also received a good deal of attention. Representative works include $[1,11,13]$.

\section{Preliminaries}

In this section, we state some definitions used throughout the remainder of the paper, many of which can be found in [4]. Some definitions are required prior to the introduction of the problem we intend to solve.

Definition 2.1. A time scale is any arbitrary nonempty closed subset of the reals. 
For our purposes of considering a boundary value problem with boundary conditions occurring at both the lower and upper extreme values of $t$ in $\mathbb{T}$, we will specify our time scale $\mathbb{T}$ as having a minimum value of 0 and a maximum value of $T$. Thus, our time scale will by default be nonempty, and we further specify our time scale $\mathbb{T}$ to be any arbitrary closed subset of $[0, T]$.

Bohner and Peterson standardized the notation for time scales in their text [4], and we include some of the more necessary definitions below.

Definition 2.2. Let $\mathbb{T}$ be a time scale. Let $t \in \mathbb{T}$. We define the forward jump operator $\sigma: \mathbb{T} \rightarrow \mathbb{T}$ by

$$
\sigma(t):=\inf \{s \in \mathbb{T}: s>t\} .
$$

We define the backward jump operator $\rho: \mathbb{T} \rightarrow \mathbb{T}$ by

$$
\rho(t):=\sup \{s \in \mathbb{T}: s<t\} .
$$

In this definition, we specify $\inf \emptyset=\sup \mathbb{T}=T$ and $\sup \emptyset=\inf \mathbb{T}=0$.

Definition 2.3. For the purpose of defining differentiation, we need to specify the time scale

$$
\mathbb{T}^{k}:=\mathbb{T}-\{T\}
$$

For the purpose of defining our boundary value problem, we need to specify the time scale

$$
\mathbb{T}^{0}:=\mathbb{T}-\{0, T\}
$$

Definition 2.4. Assume $f: \mathbb{T} \rightarrow \mathbb{R}$ is a function and let $t \in \mathbb{T}^{k}$. Then we define $f^{\Delta}(t)$ to be the number (provided it exists) with the property that given any $\varepsilon>0$, there is a neighborhood $U$ of $t$ such that

$$
\left|[f(\sigma(t))-f(s)]-f^{\Delta}(t)[\sigma(t)-s]\right| \leq \varepsilon|\sigma(t)-s|,
$$

for all $s \in U$. We call $f^{\Delta}(t)$ the delta (or Hilger) derivative of $f$ at $t$. We also make note that $f^{\Delta \Delta}(t)=\left(f^{\Delta}\right)^{\Delta}(t)$.

Having introduced these definitions, we can now introduce our problem, which will be our focus throughout this paper,

$$
u^{\Delta \Delta}(\rho(t))+f(t, u(t))=0, \quad t \in \mathbb{T}^{0},
$$

satisfying the mixed boundary conditions,

$$
u^{\Delta}(0)=u(T)=0
$$

Our goal is to prove the existence of a positive solution to this problem (2.1), (2.2) under certain assumptions concerning the function $f$ as explained below. Prior to formally defining these assumptions however, we first need a few more definitions. 
Definition 2.5. Define a solution to problem (2.1), (2.2) to mean a function $u: \mathbb{T} \rightarrow \mathbb{R}$ such that $u$ satisfies (2.1) on $\mathbb{T}^{0}$ and also satisfies the boundary conditions (2.2). If $u(t)>0$ for $t \in \mathbb{T}$, except possibly at the boundary conditions, we call $u$ a positive solution to problem (2.1), (2.2).

Definition 2.6. Let $D \subseteq \mathbb{R}$. We say $f$ is continuous on $\mathbb{T} \times D$ if $f(\cdot, x)$ is defined on $\mathbb{T}$ for each $x \in D$ and if $f(t, \cdot)$ is continuous on $D$ for each $t \in \mathbb{T}$.

Definition 2.7. Let $D \subseteq \mathbb{R}$, where $f: \mathbb{T} \times D \rightarrow \mathbb{R}$. If $D=\mathbb{R}$, then we call (2.1), (2.2) a regular problem. If $D \subsetneq \mathbb{R}$ and $f$ has singularities on the boundary of $D$, then we call (2.1), (2.2) a singular problem.

We now have sufficient definitions to introduce our first assumption (A) that will be used throughout this paper:

A: $f(t, x)$ is continuous on $\mathbb{T} \times(0, \infty)$ and $\lim _{x \rightarrow 0^{+}} f(t, x)=\infty$, for all $t \in \mathbb{T}$.

We now can specify that our problem (2.1), (2.2) is a singular boundary value problem defined on our general time scale $\mathbb{T}$. Before we can begin discussing our solution technique, however, we need to mention what we mean by integration over a general time scale $\mathbb{T}$.

Definition 2.8. A function $f: \mathbb{T} \rightarrow \mathbb{R}$ is called rd-continuous provided it is continuous at right-dense points in $\mathbb{T}$ and its left-sided limits exist (finite) at left-dense points in $\mathbb{T}$. The set of rd-continuous functions $f: \mathbb{T} \rightarrow \mathbb{R}$ will be denoted by $C_{r d}$.

Theorem 2.9. Assume $f: \mathbb{T} \rightarrow \mathbb{R}$. If $f$ is continuous, then $f$ is rd-continuous.

Theorem 2.10. Let $a, b \in \mathbb{T}$ and $f \in C_{r d}$.

1. If $\mathbb{T}=\mathbb{R}$, then

$$
\int_{a}^{b} f(t) \Delta t=\int_{a}^{b} f(t) d t
$$

where the integral on the right is the usual Riemann integral from calculus.

2. If $[a, b]$ consists only of isolated points, then

$$
\int_{a}^{b} f(t) \Delta t= \begin{cases}\sum_{t \in[a, b)} \mu(t) f(t), & \text { if } a<b \\ 0, & \text { if } a=b \\ -\sum_{t \in[b, a)} \mu(t) f(t), & \text { if } a>b\end{cases}
$$


3. If $\mathbb{T}=\mathbb{Z}$, then

$$
\int_{a}^{b} f(t) \Delta t= \begin{cases}\sum_{t=a}^{b-1} f(t), & \text { if } a<b \\ 0, & \text { if } a=b \\ -\sum_{t=b}^{a-1} f(t), & \text { if } a>b\end{cases}
$$

The previous definition and theorems are included from [4] to allow the reader a better understanding of integration on a time scale. In the next section, we relax the singular nature of (2.1), (2.2) and create a lower and upper solutions method for this similar regular problem. In the subsequent section, we use this result to a perturbation of (2.1), (2.2) so that a sequence of regular problems is created. Each of these having their own solution, we pass to a limit of this sequence, yielding the desired result of the singular problem, which we will show also satisfies the positivity condition.

\section{Lower and Upper Solutions Method}

For the purpose of establishing a lower and upper solutions method to be used in solving our pre-existing singular problem, we first consider the following regular problem,

$$
u^{\Delta \Delta}(\rho(t))+h(t, u(t))=0, \quad t \in \mathbb{T}^{0},
$$

where $h$ is continuous on $\mathbb{T} \times \mathbb{R}$ and the same boundary conditions (2.2) are satisfied. Now, (3.1), (2.2) is clearly a regular problem and it is our current goal to establish a lower and upper solutions method as a means to establish an existence result. To this end, we first must define what is meant by a lower and upper solution.

Definition 3.1. Let $\alpha: \mathbb{T} \rightarrow \mathbb{R}$. We call $\alpha$ a lower solution of problem (3.1), (2.2) if,

$$
\alpha^{\Delta \Delta}(\rho(t))+h(t, \alpha(t)) \geq 0, \quad t \in \mathbb{T}^{0},
$$

satisfying

$$
\alpha^{\Delta}(0) \geq 0, \quad \alpha(T) \leq 0 .
$$

Definition 3.2. Let $\beta: \mathbb{T} \rightarrow \mathbb{R}$. We call $\beta$ an upper solution of problem (3.1), (2.2) if,

$$
\beta^{\Delta \Delta}(\rho(t))+h(t, \beta(t)) \leq 0, \quad t \in \mathbb{T}^{0},
$$

satisfying

$$
\beta^{\Delta}(0) \leq 0, \quad \beta(T) \geq 0 .
$$

Theorem 3.3 (Lower and Upper Solutions Method). Let $\alpha$ and $\beta$ be lower and upper solutions of the regular problem (3.1), (2.2), respectively, where $\alpha \leq \beta$ on $\mathbb{T}$. Let $h(t, x)$ be continuous on $\mathbb{T} \times \mathbb{R}$. Then (3.1), (2.2) has a solution u satisfying

$$
\alpha(t) \leq u(t) \leq \beta(t), \quad t \in \mathbb{T} .
$$


Proof. We proceed with this proof through a sequence of steps involving modifications of the function $h$.

Step 1: For $t \in \mathbb{T}$ and $x \in \mathbb{R}$, define

$$
\tilde{h}(t, x)= \begin{cases}h(t, \beta(t))-\frac{x-\beta(t)}{x-\beta(t)+1}, & x>\beta(t), \\ h(t, x), & \alpha(t) \leq x \leq \beta(t), \\ h(t, \alpha(t))+\frac{\alpha(t)-x}{\alpha(t)-x+1}, & x<\alpha(t),\end{cases}
$$

Given this construction, $\tilde{h}$ is continuous on $\mathbb{T} \times \mathbb{R}$ and there exists $M>0$ so that

$$
|\tilde{h}(t, x)| \leq M
$$

for all $t \in \mathbb{T}$ and $x \in \mathbb{R}$. We now study the auxiliary equation

$$
u^{\Delta \Delta}(\rho(t))+\tilde{h}(t, u(t))=0, \quad t \in \mathbb{T}^{0},
$$

satisfying boundary conditions (2.2). Our immediate goal is to prove the existence of a solution to problem (3.7), (2.2).

Step 2: For this existence result, we lay the foundation to use the Brouwer fixed point theorem. To this end, define

$$
E=\left\{u: \mathbb{T} \rightarrow \mathbb{R} \mid u^{\Delta}(0)=u(T)=0\right\} .
$$

Also, define

$$
\|u\|=\sup \{|u(t)|: t \in \mathbb{T}\} .
$$

Given $E$ and $\|\cdot\|$, then $E$ is a Banach space. Further, we define an operator $\mathcal{T}: E \rightarrow E$ by

$$
(\mathcal{T} u)(t)=\int_{t}^{T} \int_{0}^{r} \tilde{h}(s, u(s)) \Delta s \Delta r .
$$

By construction, $\mathcal{T}$ is a continuous operator. Moreover, from the bounds placed on $\tilde{h}$ in Step 1 and from (3.8), if $r>M T^{2}$, then $\mathcal{T}(\overline{B(r)}) \subseteq \overline{B(r)}$, where $B(r):=$

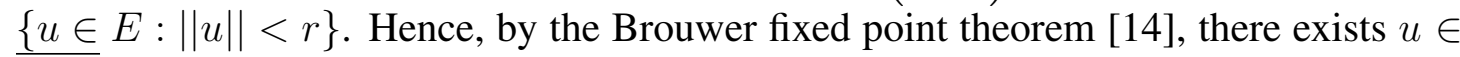
$\overline{B(r)}$ such that $u=\mathcal{T} u$.

Step 3: We now show that $u$ is a fixed point of $\mathcal{T}$ if and only if $u$ is a solution to the problem (3.7), (2.2). To this end, let us first assume that $u$ solves the problem (3.7), (2.2). Then, since the boundary conditions (2.2) are satisfied, $u \in E$. From (2.2), we also get that

$$
\int_{0}^{t} u^{\Delta \Delta}(s) \Delta s=u^{\Delta}(t)-u^{\Delta}(0)
$$




$$
=u^{\Delta}(t)
$$

and from (3.7) we conclude that

$$
u^{\Delta}(t)=-\int_{0}^{t} \tilde{h}(s, u(s)) \Delta s .
$$

Also, again from (2.2), we get that

$$
\begin{aligned}
\int_{t}^{T} u^{\Delta}(r) \Delta r & =u(T)-u(t) \\
& =-u(t)
\end{aligned}
$$

and from (3.7) we conclude that

$$
\begin{aligned}
u(t) & =-\int_{t}^{T}\left(-\int_{0}^{r} \tilde{h}(s, u(s)) \Delta s \Delta r\right) \\
& =\int_{t}^{T} \int_{0}^{r} \tilde{h}(s, u(s)) \Delta s \Delta r .
\end{aligned}
$$

Thus, $u=\mathcal{T}(u)$.

We now assume that $u$ is a fixed point of $\mathcal{T}$, i.e. $u=\mathcal{T} u$. Then $u \in E$ and satisfies (2.2). Further, since

$$
u(t)=\int_{t}^{T} \int_{0}^{r} \tilde{h}(s, u(s)) \Delta s \Delta r,
$$

we have that

$$
u^{\Delta}(t)=-\int_{0}^{t} \tilde{h}(s, u(s)) \Delta s
$$

and that

$$
u^{\Delta \Delta}(\rho(t))=-\tilde{h}(t, u(t)) .
$$

Thus, $u$ satisfies (3.7).

Step 4: The remaining piece we need to show is that solutions of (3.7), (2.2) satisfy

$$
\alpha(t) \leq u(t) \leq \beta(t), \quad t \in \mathbb{T} .
$$

To this end, without loss of generality, consider the case of obtaining $u(t) \leq \beta(t)$, and let $v(t)=u(t)-\beta(t)$. For the purpose of establishing a contradiction, assume that $\max \{v(t) \mid t \in \mathbb{T}\}:=v(l)>0$. From (2.2) and (3.5), we see that $l$ must be an interior point in $\mathbb{T}$, i.e. $l \in \mathbb{T}^{0}$. Thus, $v^{\Delta}(\rho(l)) \geq 0$ and $v^{\Delta}(l) \leq 0$, forcing $v^{\Delta \Delta}(\rho(l)) \leq 0$. Therefore,

$$
u^{\Delta \Delta}(\rho(l))-\beta^{\Delta \Delta}(\rho(l)) \leq 0 .
$$

On the other hand, we have from (3.7) and (3.6) that

$$
v^{\Delta \Delta}(\rho(l))=u^{\Delta \Delta}(\rho(l))-\beta^{\Delta \Delta}(\rho(l))
$$




$$
\begin{aligned}
& =-\tilde{h}(l, u(l))-\beta^{\Delta \Delta}(\rho(l)) \\
& =-h(l, \beta(l))+\frac{u(l)-\beta(l)}{u(l)-\beta(l)+1}-\beta^{\Delta \Delta}(\rho(l)) \\
& \geq \frac{v(l)}{v(l)+1} \\
& >0 .
\end{aligned}
$$

Hence a contradiction to (3.9) and we conclude that $\max \{v(t) \mid t \in \mathbb{T}\} \leq 0$. Hence, $v(t) \leq 0$ for all $t \in \mathbb{T}$, or rather

$$
u(t) \leq \beta(t), \quad \text { for all } t \in \mathbb{T} .
$$

A similar argument shows that $\alpha(t) \leq u(t)$ for all $t \in \mathbb{T}$.

Thus, our conclusion holds and the proof is complete.

\section{Main Result}

In this section, we make use of Theorem 3.3 to obtain positive solutions to the singular problem (2.1), (2.2). In particular, in applying Theorem 3.3, we deal with a sequence of regular perturbations of (2.1), (2.2). Ultimately, we obtain a desired solution by passing to the limit on a sequence of solutions for the perturbations.

Theorem 4.1. Assume condition (A) holds, along with the following:

B: There exists $c \in(0, \infty)$ so that $f(t, c) \leq 0$, for all $t \in \mathbb{T}^{0}$.

C: There exists $\delta>0$ so that $f(t, x)>0$ for all $t \in(T-\delta, T) \cap \mathbb{T}$ and $x \in\left(0, \frac{c}{2}\right)$.

Then, (2.1), (2.2) has a solution u satisfying

$$
0<u(t) \leq c, \quad t \in \mathbb{T}^{k}
$$

Proof. We begin by modifying our given function $f$ as follows. For $k>0, t \in \mathbb{T}$, define

$$
f_{k}(t, x)= \begin{cases}f(t,|x|), & \text { if }|x| \geq \frac{1}{k} \\ f\left(t, \frac{1}{k}\right), & \text { if }|x|<\frac{1}{k} .\end{cases}
$$

Then, $f_{k}$ is continuous on $\mathbb{T} \times \mathbb{R}$. Assumption (A) implies that there exists a $k_{0}$ such that, for all $k \geq k_{0}$,

$$
f_{k}(t, 0)=f\left(t, \frac{1}{k}\right)>0, \quad \text { for all } t \in \mathbb{T}
$$


We now consider the boundary value problem

$$
u^{\Delta \Delta}(\rho(t))+f_{k}(t, u(t))=0, \quad t \in \mathbb{T}^{0},
$$

satisfying boundary conditions (2.2).

Now, let $\alpha(t)=0$ and $\beta(t)=c$. Then, for each $k \geq k_{0}, \alpha$ and $\beta$ are lower and upper solutions of (4.1), (2.2), respectively. Also, $\alpha(t) \leq \beta(t)$ for $t \in \mathbb{T}$. Thus, by Theorem 3.3, for each $k \geq k_{0}$, there exists a solution $u_{k}$ to each problem (4.1), (2.2) that satisfies $0 \leq u_{k}(t) \leq c$, for $t \in \mathbb{T}$.

Let $\mathbb{T}_{1}=\mathbb{T} \cap(0, T-\delta)$ and $\mathbb{T}_{2}=\mathbb{T} \cap(T-\delta, T)$.

Now, there exists $\varepsilon \in\left(0, \frac{c}{2}\right)$ such that if $k \geq k_{\varepsilon} \geq k_{0}$, then

$$
f_{k}(t, x)>c, \quad t \in \mathbb{T}, x \in(0, \varepsilon] .
$$

For the sake of establishing a contradiction, assume that for $k \geq k_{\varepsilon} \geq k_{0}$, we have that $u_{k_{\varepsilon}}(t)<\varepsilon_{1}$, where

$$
\varepsilon_{1}= \begin{cases}\varepsilon, & t \in \mathbb{T}_{1} \\ \frac{\varepsilon}{\delta}(T-t), & t \in \mathbb{T}_{2}\end{cases}
$$

Now,

$$
\begin{aligned}
-u_{k_{\varepsilon}}(t)= & \int_{t}^{T} u_{k_{\varepsilon}}^{\Delta}(r) \Delta r \\
= & -\int_{t}^{T} \int_{0}^{r} f_{k_{\varepsilon}}\left(s, u_{k_{\varepsilon}}(s)\right) \Delta s \Delta r \\
= & -\int_{t}^{T-\delta} \int_{0}^{r} f_{k_{\varepsilon}}\left(s, u_{k_{\varepsilon}}(s)\right) \Delta s \Delta r \\
& -\int_{T-\delta}^{T} \int_{0}^{r} f_{k_{\varepsilon}}\left(s, u_{k_{\varepsilon}}(s)\right) \Delta s \Delta r \\
< & -\int_{T-\delta}^{T} \int_{0}^{r} c \Delta s \Delta r \\
= & -\frac{c}{2}\left((T-\delta)^{2}-t^{2}\right) .
\end{aligned}
$$

First, consider $t \in \mathbb{T}_{1}$. We have that $u_{k_{\varepsilon}}(t)>\frac{c}{2}\left((T-\delta)^{2}-t^{2}\right)$. However, this implies that $u_{k_{\varepsilon}}^{\Delta \Delta}(\rho(t))>-c$, which leads to $f_{k_{\varepsilon}}\left(t, u_{k_{\varepsilon}}(t)\right)<c$, a contradiction to (4.2). Hence, for $t \in \mathbb{T}_{1}$, we have

$$
u_{k_{\varepsilon}}(t) \geq \varepsilon_{1}=\varepsilon
$$

Now, consider $t \in \mathbb{T}_{2}$. We have that $u_{k_{\varepsilon}}>\frac{c}{2}\left((T-\delta)^{2}-t^{2}\right)$. This again implies that $u_{k_{\varepsilon}}^{\Delta \Delta}(\rho(t))>-c$. We also have, from assumption $(\mathbf{C})$, that $u_{k_{\varepsilon}}^{\Delta \Delta}(\rho(t))=-f_{k_{\varepsilon}}\left(t, u_{k_{\varepsilon}}(t)\right)<$ 
0. Hence, $-c<u_{k_{\varepsilon}}^{\Delta \Delta}(\rho(t))<0$, making $u_{k_{\varepsilon}}$ concave in this interval. We also know, by continuity, that $u_{k_{\varepsilon}}(T-\delta) \geq \varepsilon$ and $u_{k_{\varepsilon}}(T)=0$. Therefore, by continuity, for $t \in \mathbb{T}_{2}$,

$$
u_{k_{\varepsilon}}(t)>\varepsilon_{1}=\frac{\varepsilon}{\delta}(T-t) .
$$

Hence, $0<\varepsilon_{1}<u_{k_{\varepsilon}}(t)$ for $t \in \mathbb{T}^{0}$. Thus, for $k \geq k_{\varepsilon}$, we can choose a subsequence $\left\{u_{k_{n}}(t)\right\} \subseteq\left\{u_{k}(t)\right\}$ so that

$$
\lim _{n \rightarrow \infty} u_{k_{n}}(t)=u(t), \quad t \in \mathbb{T},
$$

and note that $u(t) \in E$, where $E$ is defined as in the proof of Theorem 3.3.

Moreover, for sufficiently large $n$,

$$
u_{k_{n}}=\int_{t}^{T} \int_{0}^{r} f\left(s, u_{k_{n}}(s)\right) \Delta s \Delta r
$$

And from the continuity of $f$, as we let $n \rightarrow \infty$, we get

$$
u(t)=\int_{t}^{T} \int_{0}^{r} f(s, u(s)) \Delta s \Delta r
$$

Hence,

$$
u^{\Delta \Delta}(\rho(t))=-f(t, u(t)),
$$

with the desired inequality satisfied, specifically, $0<u(t) \leq c$ on $\mathbb{T}^{k}$.

\section{Example}

Let $\mathbb{T}$ be as given following Definition 2.1. Let $\alpha \in[0, \infty), c, \beta \in(0, \infty)$, and $a: \mathbb{T} \rightarrow$ $\mathbb{R}$. Then, by Theorem 4.1 , the problem

$$
u^{\Delta \Delta}(\rho(t))+\left(a(t)+(u(t))^{\alpha}+(u(t))^{-\beta}\right)(c-u(t))-\left(u^{\Delta}(\rho(t))\right)^{3}=0, \quad t \in \mathbb{T}^{k},
$$

along with the boundary conditions (2.2), has a solution $u$ satisfying the desired inequality. It is worth noting that although the function $f$ in problem (2.1) does not depend on $u^{\Delta}(\rho(t))$ explicitly, $u^{\Delta}(\rho(t))$ is well defined on $\mathbb{T}^{k}$ and in many cases can be rewritten simply in terms of $u(t)$.

\section{References}

[1] R. P. Agarwal and D. O'Regan. Singular discrete boundary value problems. Appl. Math. Lett., 12(4):127-131, 1999. 
[2] Samerah Al Mosa and Paul Eloe. Upper and lower solution method for boundary value problems at resonance. Electron. J. Qual. Theory Differ. Equ., pages Paper No. 40, 13, 2016.

[3] Gui Bao, Xian Xu, and Yan Song. Positive solutions for three-point boundary value problems with a non-well-ordered upper and lower solution condition. Appl. Math. Lett., 25(4):767-770, 2012.

[4] Martin Bohner and Allan Peterson. Dynamic equations on time scales. Birkhäuser Boston, Inc., Boston, MA, 2001. An introduction with applications.

[5] Josef Diblík and Jiří Vítovec. Lower and upper estimates of solutions to systems of delay dynamic equations on time scales. Bound. Value Probl., pages 2013:260, $14,2013$.

[6] Robert Hakl and Manuel Zamora. Existence of a solution to the Dirichlet problem associated to a second-order differential equation with singularities: the method of lower and upper functions. Georgian Math. J., 20(3):469-491, 2013.

[7] Johnny Henderson and Curtis J. Kunkel. Singular discrete higher order boundary value problems. Int. J. Difference Equ., 1(1):119-133, 2006.

[8] Curtis J. Kunkel. Singular second order boundary value problems for differential equations. In Proceedings of Neural, Parallel, and Scientific Computations. Vol. 3, pages 119-124. Dynamic, Atlanta, GA, 2006.

[9] Curtis J. Kunkel. Singular second order boundary value problems on purely discrete time scales. J. Difference Equ. Appl., 14(4):411-420, 2008.

[10] K. R. Prasad, P. Murali, and N. V. V. S. Suryanarayana. Upper and lower solutions for general two-point boundary value problems on time scales. Neural Parallel Sci. Comput., 21(1):31-41, 2013.

[11] Radu Precup. Abstract method of upper and lower solutions and application to singular boundary value problems. Stud. Univ. Babeş-Bolyai Math., 61(4):443$451,2016$.

[12] Irena Rachunková and Lukáš Rachunek. Singular discrete second order BVPs with p-Laplacian. J. Difference Equ. Appl., 12(8):811-819, 2006.

[13] Irena Rachunková and Lukáš Rachunek. Singular discrete and continuous mixed boundary value problems. Math. Comput. Modelling, 49(3-4):413-422, 2009.

[14] Eberhard Zeidler. Nonlinear functional analysis and its applications. I. SpringerVerlag, New York, 1986. Fixed-point theorems, Translated from the German by Peter R. Wadsack. 
